Journal for ImmunoTherapy of Cancer

\title{
Neoadjuvant immunoradiotherapy results in high rate of complete pathological response and clinical to pathological downstaging in locally advanced head and neck squamous cell carcinoma
}

Rom Leidner (10 ,1,2 Marka Crittenden, ${ }^{1,2,3}$ Kristina Young, ${ }^{1,2,3}$ Hong Xiao, ${ }^{4}$ Yaping Wu, ${ }^{4}$ Marcus A Couey, ${ }^{1}$ Ashish A Patel, ${ }^{1,5}$ Allen C Cheng, ${ }^{5}$ Amber L Watters, ${ }^{1}$ Carlo Bifulco, ${ }^{1,2,4}$ George Morris, ${ }^{2}$ Lessli Rushforth, ${ }^{2}$ Shorin Nemeth, ${ }^{1}$ Walter J Urba, ${ }^{1,2}$ Michael Gough (1) , ${ }^{1,2}$ R Bryan Bell (D) ${ }^{1,2}$

To cite: Leidner $\mathrm{R}$, Crittenden $\mathrm{M}$, Young $\mathrm{K}$, et al. Neoadjuvant immunoradiotherapy results in high rate of complete pathological response and clinical to pathological downstaging in locally advanced head and neck squamous cell carcinoma. Journal for ImmunoTherapy of Cancer 2021;9:e002485. doi:10.1136/ jitc-2021-002485

- Additional material is published online only. To view, please visit the journal online (http://dx.doi.org/10.1136/jitc2021-002485).

Accepted 09 March 2021

Check for updates

(C) Author(s) (or their employer(s)) 2021. Re-use permitted under CC BY-NC. No commercial re-use. See rights and permissions. Published by BMJ.

For numbered affiliations see end of article.

Correspondence to

Dr R Bryan Bell;

richard.bell@providence.org

\section{ABSTRACT}

Background Checkpoint inhibitors targeting programmed death receptor-1 (PD-1) have been tested in the neoadjuvant setting for the treatment of locoregionally advanced head and neck squamous cell carcinoma (HNSCC); however, response rates are modest. We hypothesized that adding stereotactic body radiation therapy (SBRT) to anti-PD-1 would be safe prior to definitive surgical resection and would enhance pathological response compared with historical cohorts of patients with locoregionally advanced HNSCC treated with checkpoint inhibitor alone.

Methods The Neoadjuvant Immuno-Radiotherapy Trial was an investigator-initiated single institution phase Ib clinical trial that enrolled patients with previously untreated locally advanced HPV-positive and HPV-negative HNSCC between 2018 and 2019. Eligible patients were treated with neoadjuvant SBRT at a total dose of either 40 Gy in 5 fractions or 24 Gy in 3 fractions, delivered in a 1-week timespan, with or without nivolumab, prior to definitive surgical resection. Patients were then planned for treatment with adjuvant nivolumab for 3 months. The primary safety endpoint was unplanned delay in surgery considered to be at least possibly related to neoadjuvant treatment. The primary efficacy endpoints included pathological complete response (pCR), major pathological response $(\mathrm{mPR})$, and the rate of clinical to pathological downstaging after neoadjuvant treatment.

Results Twenty-one patients underwent neoadjuvant treatment, which was well tolerated and did not delay surgery, thus meeting the primary endpoint. Tissue responses were characterized by robust inflammatory infiltrates in the regression bed, plasma cells and cholesterol clefts. Among the entire study group, the mPR and $\mathrm{pCR}$ rate was $86 \%$ and $67 \%$, respectively. Clinical to pathological downstaging occurred in $90 \%$ of the patients treated.

Conclusion These data demonstrate that radiation delivered only to the gross tumor volume combined with immunotherapy was safe, resulted in a high rate of $\mathrm{mPR}$ and should be further evaluated as a locally focused neoadjuvant therapy for patients with head and neck cancer.

Trial registration number This study is registered with clinicaltrials.gov (NCT03247712) and is active, but closed to patient accrual.

\section{INTRODUCTION}

Neoadjuvant immunotherapy with checkpoint inhibitors (CI) targeting programmed death receptor-1 (PD-1) have resulted clinically meaningful pathological responses and clinical to pathological downstaging in multiple solid tumor types. ${ }^{1-5}$ In high-risk resectable stage III/IV melanoma, complete or major pathological response (mPR) to PD-1 blockade, defined as $<10 \%$ viable tumor cells in the regression bed, occurs in $30 \%$ of patients and has been associated with improved disease-free survival, the accumulation of tumor-infiltrating lymphocytes, increased $\mathrm{T}$ cell clonality and broadened $\mathrm{T}$ cell repertoire. ${ }^{2}$ In head and neck squamous cell carcinoma (HNSCC), however, mPR to neoadjuvant CI (nivolumab or pembrolizumab) occurs in only $7 \%-14 \%$ of patients with human papilloma virus (HPV)-unrelated tumors, whether treated with monotherapy ${ }^{67}$ or in combination with ipilimuab. ${ }^{8}$ Among patients with locally advanced HPV-related oropharyngeal squamous cell carcinoma, the $\mathrm{mPR}$ rate to durvalumab or durvalumab plus tremelimumab is as high as $29 \% .^{9}$

Locally advanced HNSCC is treated with either surgical resection of the primary and 
draining lymph nodes followed by pathological riskadapted adjuvant radiation $(60 \mathrm{~Gy} / 30$ fractions $)$, with or without chemotherapy (cisplatin $100 \mathrm{mg} / \mathrm{m}^{2}$ once every 3 weeks; or weekly $\left.40 \mathrm{mg} / \mathrm{m}^{2}\right)$; or cisplatin $(100 \mathrm{mg}$ / $\left.\mathrm{m}^{2}\right)$ every 3 weeks or weekly cisplatin $\left(40 \mathrm{mg} / \mathrm{m}^{2}\right)$ with concurrent radiotherapy (RT) using standard once time a day fractionation of $70 \mathrm{~Gy} / 35$ fractions, which can be a morbid approach that is associated with a $67 \%-81 \%$ rate of grade 3-4 adverse events (AEs). ${ }^{10-12}$ Neoadjuvant immunotherapy has the potential to enhance local control and quality of life through immune mediated cytoreduction and reduced tumor volume to facilitate more limited surgery, as well deintensify or eliminate the need for adjuvant therapy. ${ }^{13}$ To improve response rates, there has recently been a surge of interest in combining immunotherapy with stereotactic body radiation therapy (SBRT). ${ }^{1415}$ The rationale for these studies in the metastatic setting is that the addition of radiation will incrementally improve the systemic response seen with PD-1 blockade and other immunotherapies, via a radiation in-situ vaccination effect, to drive a systemic antitumor immune response beyond the irradiated field (abscopal effect) ${ }^{16}$ While randomized clinical trials have not shown distant tumor responses with RT and CI to be different from those seen with CI alone, ${ }^{17} 18$ there is preclinical evidence to suggest that the combination may exert a far greater influence on local disease control by manipulating the phenotype of cancer cells in the treatment field and rendering them more susceptible to immune killing. ${ }^{19-24}$

It has been shown that PD-L1 upregulation following radiation can limit local control of tumors in murine models and that blockade of the PD-1/PD-L1 axis concurrent with radiation results in enhanced tumor control. ${ }^{22}$ Notably, conventional linear-quadratic models of cell killing may not accurately predict response at higher dose ranges as they do not account for the tumor microenvironment, consisting of vasculature, immune and stromal cells, which are implicated in RT failure, but conversely may contribute to improved local control when appropriate immune targeting agents are combined. ${ }^{25-27}$ Furthermore, it has been demonstrated that elective nodal irradiation reduces the efficacy of combination stereotactic radiation therapy and immunotherapy. ${ }^{28}$ Given the significant acute morbidity associated with 6-7 weeks of definitive chemoradiation, or 6 weeks of adjuvant radiation \pm chemotherapy, and the critical importance of local control in head and neck cancer, the neoadjuvant setting may be ideal for assessing a therapeutically beneficial interaction between SBRT and PD-1 blockade. In addition, neoadjuvant immunoradiation has the potential to enhance quality of life by shortening the duration of treatment, limiting radiation fields, facilitating less extensive surgery through downstaging, and eliminating adjuvant therapy in some patients.

Based on this rationale, we propose that neoadjuvant PD-1 blockade in combination with SBRT would be safe, would not delay definitive surgical resection, and would result in a greater than $33 \% \mathrm{mPR}$ and clinical to pathological downstaging. Here, we report the results of a first in human phase Ib trial of neoadjuvant SBRT delivered to gross tumor volume (GTV) in combination with nivolumab prior to surgery for curative intent and adjuvant nivolumab in patients with locoregionally advanced HNSCC (NCT03247712).

\section{METHODS \\ Study population and trial design}

The Neoadjuvant Immuno-Radiotherapy Trial (NIRT) was an investigator-initiated single institution phase Ib clinical trial sponsored by Providence Health and Services-Oregon that enrolled patients with previously untreated locally advanced p16-positive (stages I-III, American Joint Committee on Cancer (AJCC) Cancer Staging Manual, 8th Ed) and p16-negative (stages IIIIVA) HNSCC. Initially, patients were enrolled to one of two dose-finding cohorts to undergo neoadjuvant SBRT delivered to GTV only over 1 week at a total dose of either $40 \mathrm{~Gy}$ in 5 fractions, Monday-Friday (cohort 1), or 24 Gy in 3 fractions, Monday-Wednesday-Friday (cohort 2), in combination with nivolumab $240 \mathrm{mg}$ intravenous $\mathrm{q} 2$ weeks $\times 3$ prior to surgery (figure 1 ). After observing for dose-limiting toxicity in the safety portion of the trial, we added two expansion cohorts that evaluated neoadjuvant treatment at the lower radiation dose $(24 \mathrm{~Gy}, 8 \mathrm{~Gy}$ X3) with and without immunotherapy: cohort 3 consisted of patients with stages I-III HPV+ HNSCCwho were treated with SBRT alone in the neoadjuvant phase to act as a control; patients in cohort 4 had stages III-IVA p16negative HNSCC and were treated with nivolumab and SBRT as in cohort 2. Surgery in all cohorts was planned 5 weeks post SBRT, followed by adjuvant nivolumab $480 \mathrm{mg}$ intravenous q 4 weeks for 3 doses starting 4 weeks after surgery.

All patients provided written informed consent. Patients were eligible for enrollment if they were age 18 years or older, had an Eastern Cooperative Oncology Group performance status deemed suitable by investigator for study requirement, and had adequate organ function to meet protocol criteria. Patients had to be considered by the investigators to be candidates for surgery and adjuvant radiation as part of standard of care. Patients with bulky primary tumor, extensive neck disease or radiographic evidence of extra nodal extension were allowed, but patients who in the opinion of the investigators could likely be treated with surgery alone or for which radiation would not have been indicated during a standard treatment course were excluded. Patients who might have been treated with primary chemoradiation for organ preservation but in whom data supports upfront surgical resection, such as cartilage-invading T4 larynx cancer, were allowed. The radiation technique is detailed in online supplemental file 1 . The target lesions (primary tumor and all radiographically apparent metastatic lymph nodes) were outlined and confirmed by two 


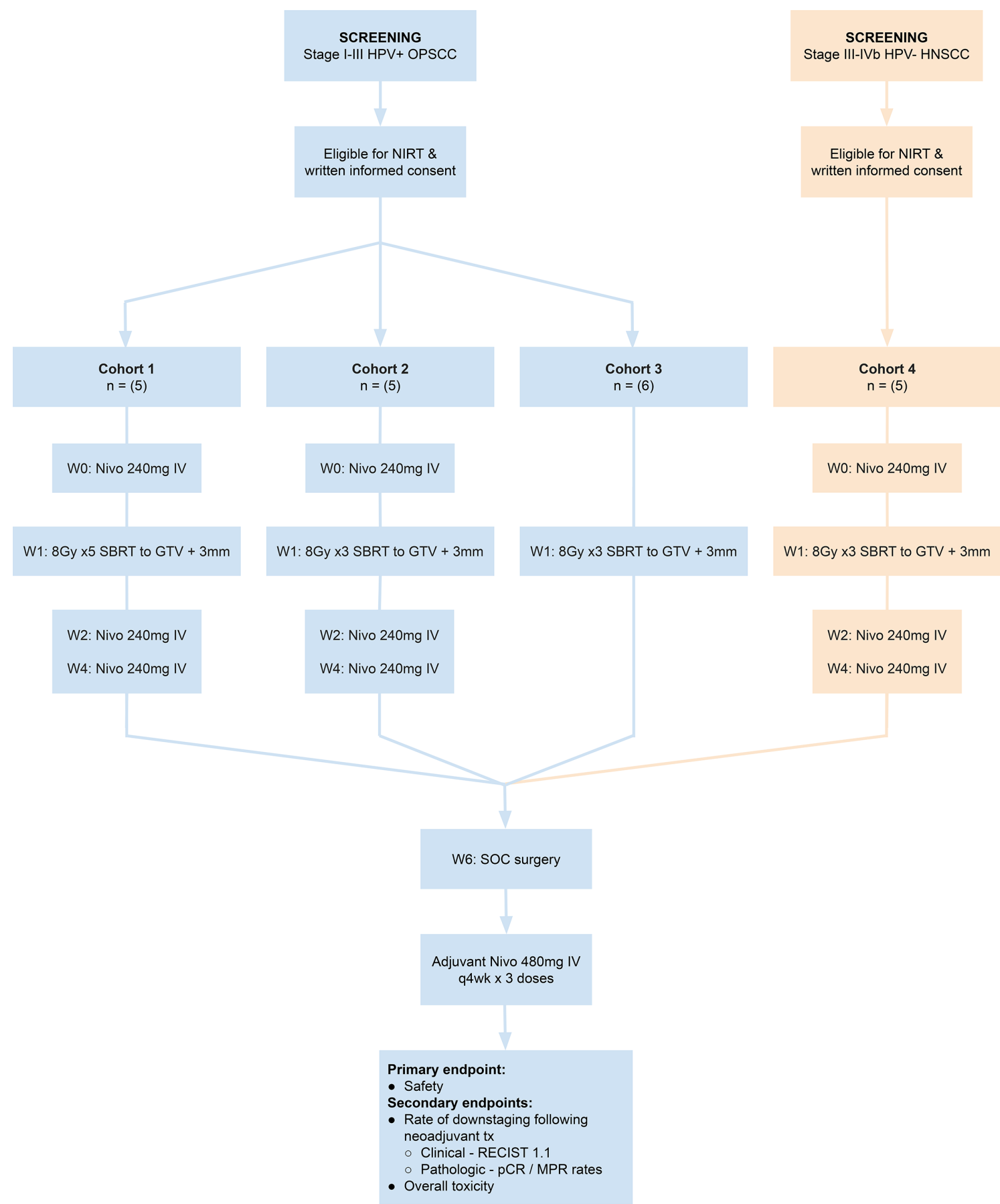

Figure 1 Neoadjuvant Immuno-Radiotherapy Trial (NIRT) schema. GTV, gross tumor volume; HNSCC, head and neck squamous cell carcinoma; HPV, human papilloma virus; IV, intravenous; MPR, major pathological response; pCR, pathological complete response; SBRT, stereotactic body radiation therapy; SOC, standard of care.

radiation oncologists (MRC, $\mathrm{KHY}$ ) and designated as GTV. The GTV was expanded uniformly by an additional 2-3 $\mathrm{mm}$ to create a planning target volume. Radiation was delivered 1 week after the first nivolumab dose (for cohorts 1, 2 and 4). Surgery was planned 5 weeks after radiation and consisted of neck dissection (levels II-IV for pharynx and larynx; I-IV or I-V for oral cavity) in combination with transoral robotic assisted oropharyngectomy or composite resection as appropriate for patients with mucosal disease. Resection of the primary was performed in enbloc fashion to clear clinically abnormal tissue in the regression bed with a $1 \mathrm{~cm}$ margin of normal appearing tissue, not to preradiation tumor margins. The protocol called for standard histopathological risk-adapted adjuvant RT for patients with T3/4 tumors, perineural invasion, lymphovascular invasion, or $>1$ metastatic lymph nodes. Patients with positive resection margins or $>1 \mathrm{~mm}$ extracapsular extension in a metastatic lymph node were to receive adjuvant chemoradiation. Determination for adjuvant treatment was based on the findings at the time 
of surgery following neoadjuvant therapy and not based on pretreatment staging. Following standard of care, all patients were planned to receive adjuvant nivolumab $480 \mathrm{mg}$ intravenous every 4 weeks for 3 months.

All patients had a tumor biopsy at baseline and 1 week following radiation, with paired blood samples at pretreatment and various post-treatment time points. The biopsies and resection specimens were processed in the Department of Pathology at Providence Portland Medical Center, Providence Health and Services, and immediately formalin fixed and paraffin embedded. Baseline imaging consisted of at least a contrasted CT of the neck and chest as well as positive emission tomography (PET)/CT within 30 days of the therapy with a restaging neck CT the week prior to surgery. Follow-up and imaging was planned for every 3-6 months per standard of care.

The primary safety endpoint was unplanned delay in surgery considered to be at least possibly related to neoadjuvant treatment. Neoadjuvant treatment would be considered safe if there were two or fewer delays among the first six patients enrolled. Early stopping rules (Section 6.7 in protocol) (online supplemental material 1) of this trial were developed based on unacceptable toxicity as assessed using Common Toxicity Criteria for Adverse Events (CTCAE) scale version 4, and the ClavienDindo grading tool for surgical complications. ${ }^{29}$ DLT were based on unacceptable AEs, defined as either an unplanned delay in surgery or grade 3 or $4 \mathrm{AE}$ per the CTCAE or the Clavien-Dindo grading system for surgical complications.

The primary efficacy endpoints were pathological response and clinical to pathological downstaging after neoadjuvant treatment. Pathological response in the primary lesion and metastatic lymph node(s) were quantified by a head and neck pathologist as percentages of viable tumor versus areas showing nonviable tumor and/or evidence of tissue response to non-viable tumor observed on $\mathrm{H} \& \mathrm{E}$ staining of the resection specimen, as described by Cottrell et al. ${ }^{80} \mathrm{cPR}$ was defined as the absence of viable tumor cells. mPR was defined as fewer than $10 \%$ viable tumor cells. Clinical to pathological downstaging was assessed using AJCC eighth edition staging manual. Secondary end points included objective radiographic response prior to surgery using RECIST 1.1 criteria and gastrostomy tube dependence.

\section{Statistical analysis}

In addition to safety, the goal of this study was to identify clinical to pathological downstaging and mPR in $33 \%$ or more patients using Simon's two-stage design (optimal). This design yields an alpha $=0.05$ level of significance with a power of $90 \%$ to detect a difference when the true rate of downstaging $\geq 33 \%$. Using these criteria, the study was stopped when there was sufficient evidence that the mPR exceeded $33 \%$ in all cohorts. The null hypothesis, that the true rate of downstaging is $\leq 10 \%$, was tested against a one-sided alternative. If 1 or fewer downstaging events were observed for the first 12 patients enrolled, the study would be stopped early for futility. Analyses were performed using SAS statistical software (V.9.4; SAS Institute) and JMP Pro statistical software (V.15.0; SAS Institute) based on database as of 15 July 2020.

\section{RESULTS}

\section{Patient characteristics}

From February 2018 through November 2019, we enrolled 24 patients, 3 of whom were ineligible for inclusion and did not receive treatment, leaving 21 patients (table 1). Initially, patients were enrolled sequentially to the first two cohorts. After safety and efficacy analysis, cohorts 3 and 4 were opened simultaneously. The original protocol called for 28 patients; however, the study was closed to enrollment after safety was established and efficacy endpoints met statistical criteria as a positive study. Demographic characteristics are listed in table 1: $67 \%$ had primary tumor located in the oropharynx, $9.5 \%$ in the oral cavity, $9.5 \%$ in the larynx and $14 \%$ had unknown primaries. Of 21 patients, $16(76 \%)$ had HPV-positive disease as determined by p16 staining (stage 1 or 2 , AJCC 8th Ed) and all 5 of the HPV-negative patients had locoregionally advanced stage IVA disease, two of which had cartilage-invading T4 larynx cancer.

\section{Safety outcomes}

All 21 patients completed neoadjuvant treatment and there were no dose-limiting toxicities in the safety run-in portion of the study. The mean time to surgery from first nivolumab dose was 44.6 days (range $=40-54$ days) and there were no treatment-related surgical delays in any of the 21 patients enrolled, thus meeting the primary safety endpoint. Treatment-related AE's (TRAE) of any grade at least possibly related to neoadjuvant therapy occurred in all patients and most commonly consisted of mucositis $(\mathrm{n}=20,95 \%)$, thrush/dysgeusia/xerostomia $(\mathrm{n}=15,71 \%), \mathrm{rash} /$ pruritus $(\mathrm{n}=11,52 \%)$, head and neck pain $(n=9,43 \%)$, fatigue $(n=8,38 \%)$, hypotension $(n=6$, $29 \%)$, nausea/vomiting $(\mathrm{n}=4,19 \%)$ and anemia $(\mathrm{n}=4$, $19 \%$; table 2). Grade 3 TRAE's attributed to neoadjuvant therapy included mucositis $(\mathrm{n}=1,5 \%)$, anemia $(\mathrm{n}=1,5 \%)$, hypotension $(\mathrm{n}=1,5 \%)$ and hyponatremia $(\mathrm{n}=1,5 \%)$.

In general, surgery was uncomplicated, although submucosal tissue planes were subjectively less well defined and more inflamed in patients who received the higher radiation dose (cohort 1). Although not an endpoint in the study, surgical resection of the primary tumor was reduced in most patients and was performed to the margins observed after neoadjuvant therapy, not the original tumor margins. Surgical complications were assessed by Clavien-Dindo scoring and included most commonly head and neck pain $(\mathrm{n}=9,43 \%)$, nausea/ vomiting $(\mathrm{n}=8,38 \%)$, delayed wound healing/dehiscence $(\mathrm{n}=7,33 \%)$, cough/increased secretions $(\mathrm{n}=6$, $29 \%)$, hematoma/seroma ( $\mathrm{n}=5,33 \%)$, anxiety/disorientation $(\mathrm{n}=5,24 \%)$ and neurosensory changes $(\mathrm{n}=5,24 \%)$ (table 2). Postoperative complications that required 
Table 1 Baseline characteristics of the 21 study patients

No. (\%)

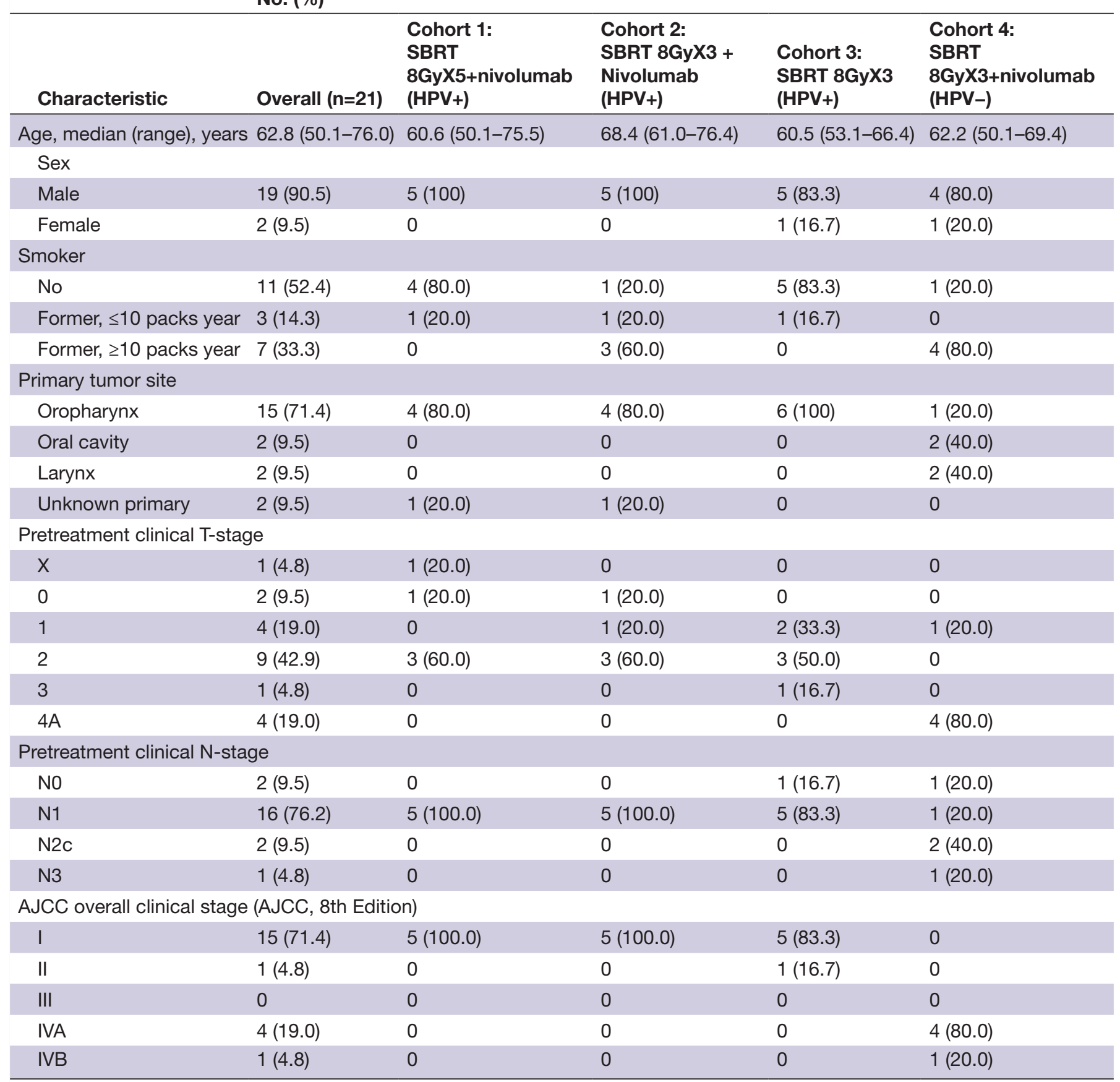

HPV, human papilloma virus; SBRT, stereotactic body radiation therapy.

surgical intervention, included hematoma/seroma that required drainage $(n=3,14 \%)$, delayed wound healing/ dehiscence requiring debridement $(n=2,10 \%)$, weight loss resulting in feeding tube placement $(n=1,5 \%)$, pneumothorax and pneumonia requiring chest tube placement $(n=1,5 \%)$, and urinary retention requiring indwelling catheter placement $(n=1,5 \%)$.

There were two types of postoperative wound healing problems, which were grouped together for practical purposes: delayed wound healing and wound dehiscence. Delayed wound healing was observed at the primary mucosal resection site in three patients with oropharyngeal cancer who underwent transoral robotic surgery, was manifested as superficial tissue necrosis, and thought to be due to a combination of all three treatment modalities. All of these patients healed uneventfully, although one underwent operative debridement of necrotic tissue in the wound bed. Wound dehiscence at the flap/ mucosal resection margin was observed in four patients in cohort 4, all of whom had undergone major composite tissue resection and free flap reconstruction. Three of the patients had minor wound dehiscence that healed 


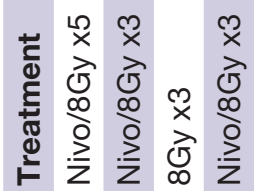
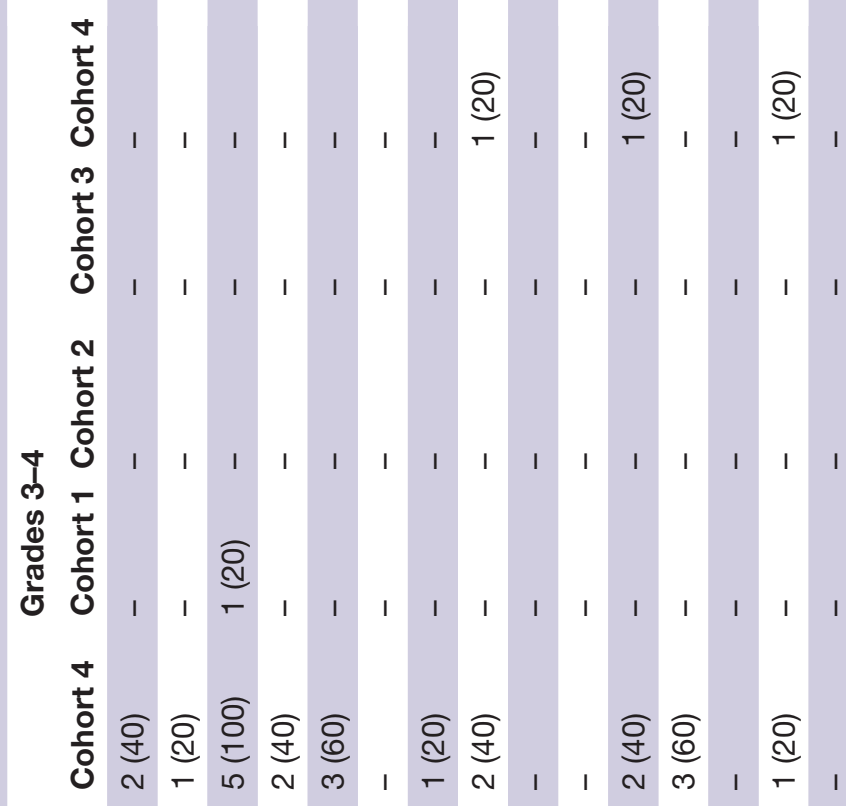

$\stackrel{m}{t}$

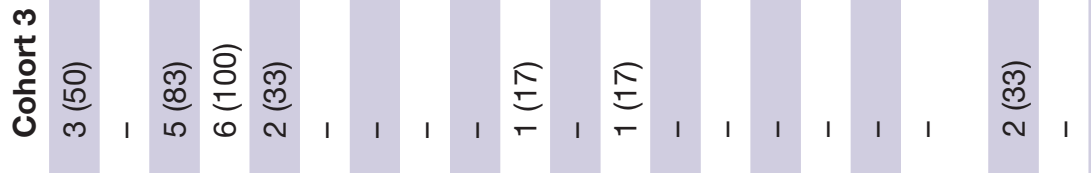

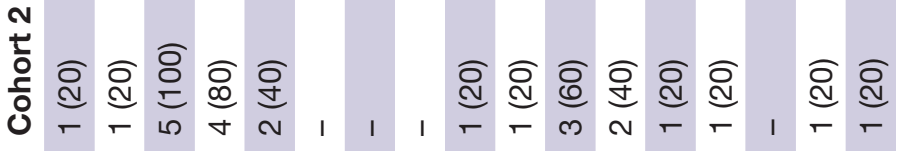

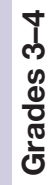

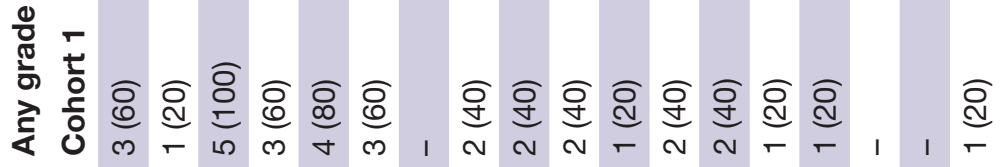

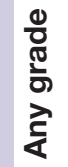

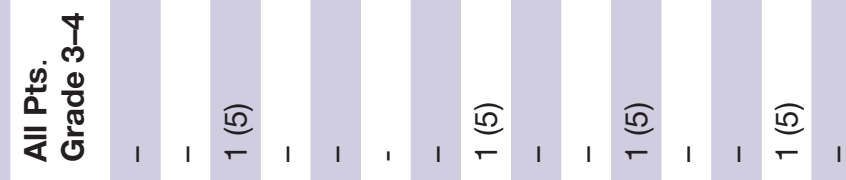

을 贾

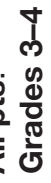

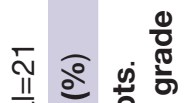

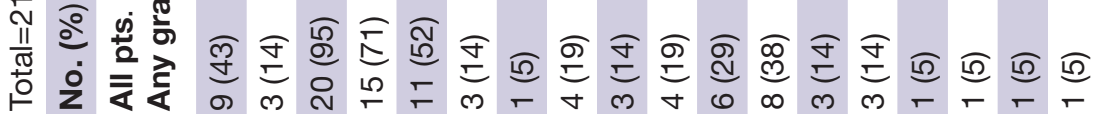

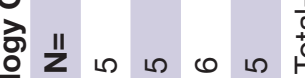

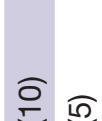

$\frac{0}{\frac{6}{0}} \frac{\pi}{2}$ 


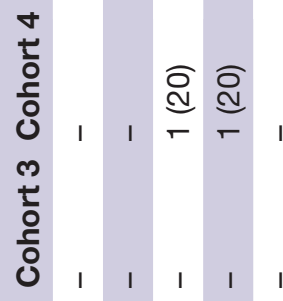

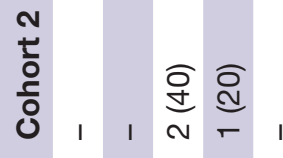

产

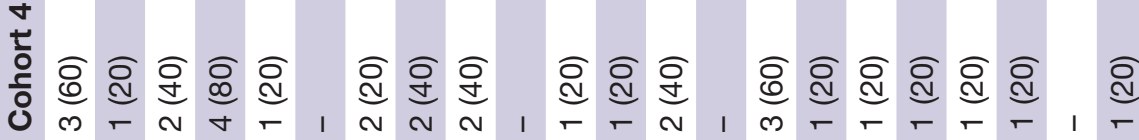

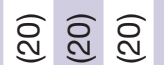

Ф્

बे

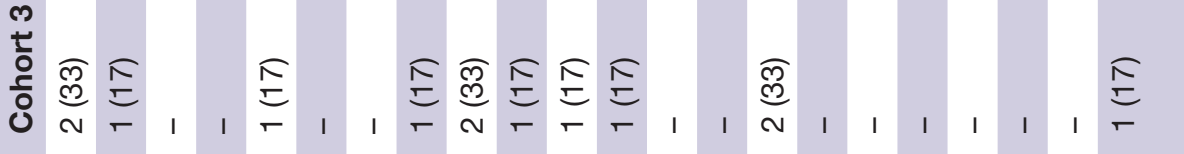

N

范

产

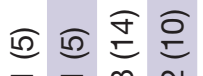

ఏ)

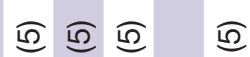

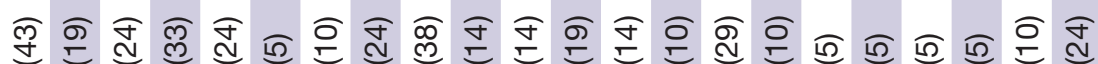

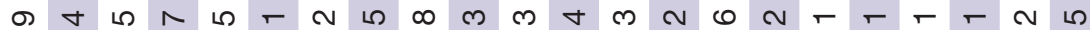
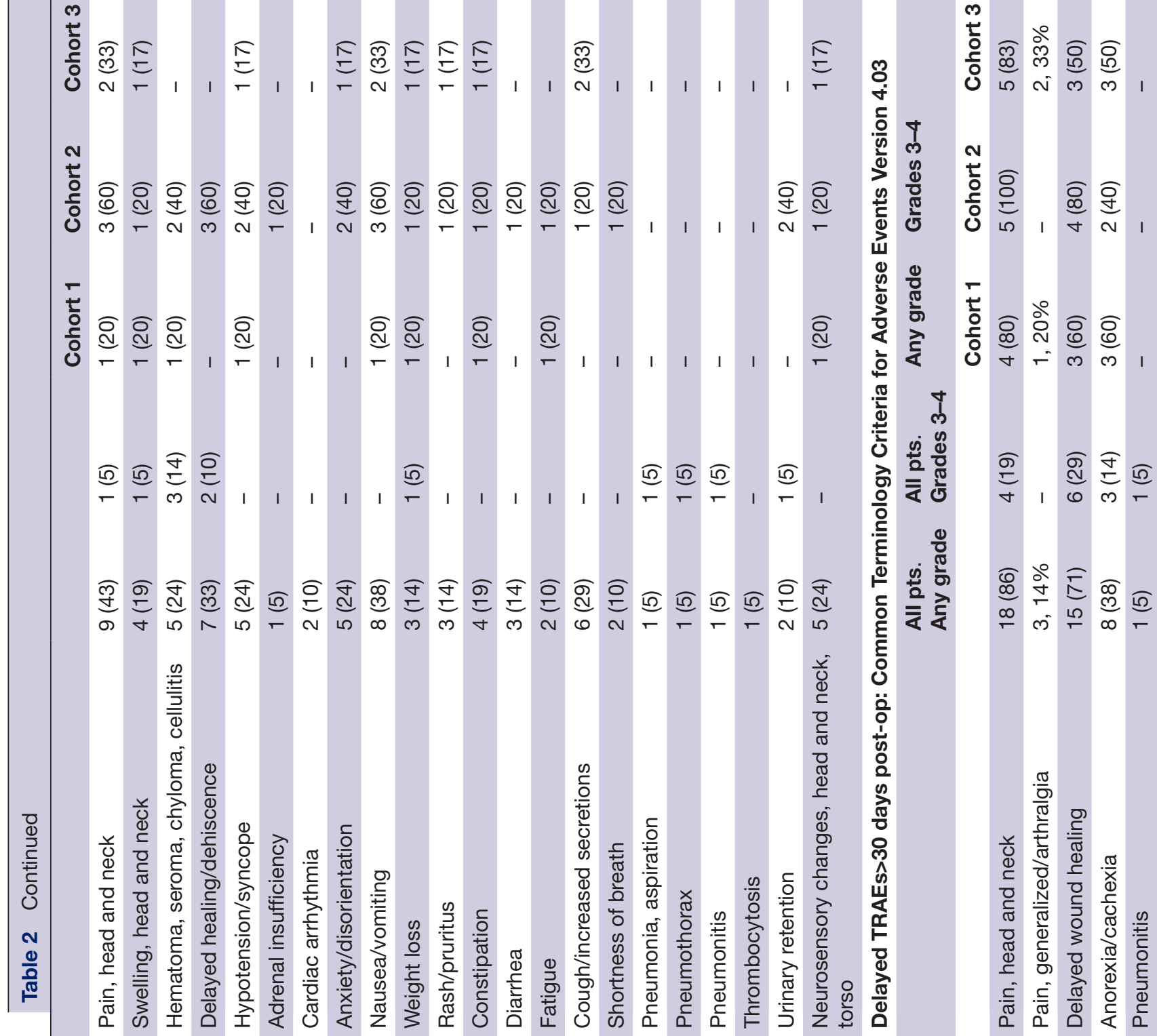

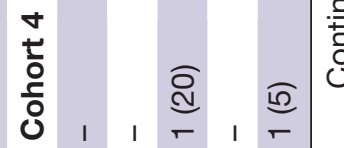

足

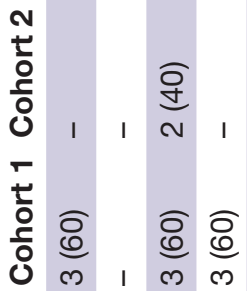

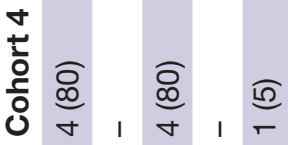

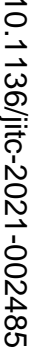

@

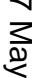

恣

ڤั

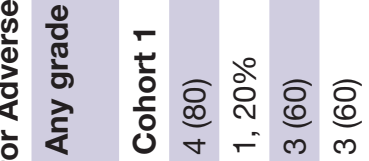

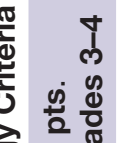

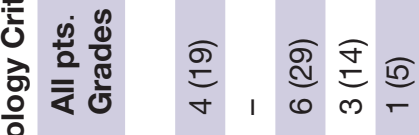

旁.

ᄋ

을.

N

N

एव:

$\stackrel{0}{\overline{0}}$

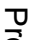

융

$\stackrel{2}{\circ}$ 


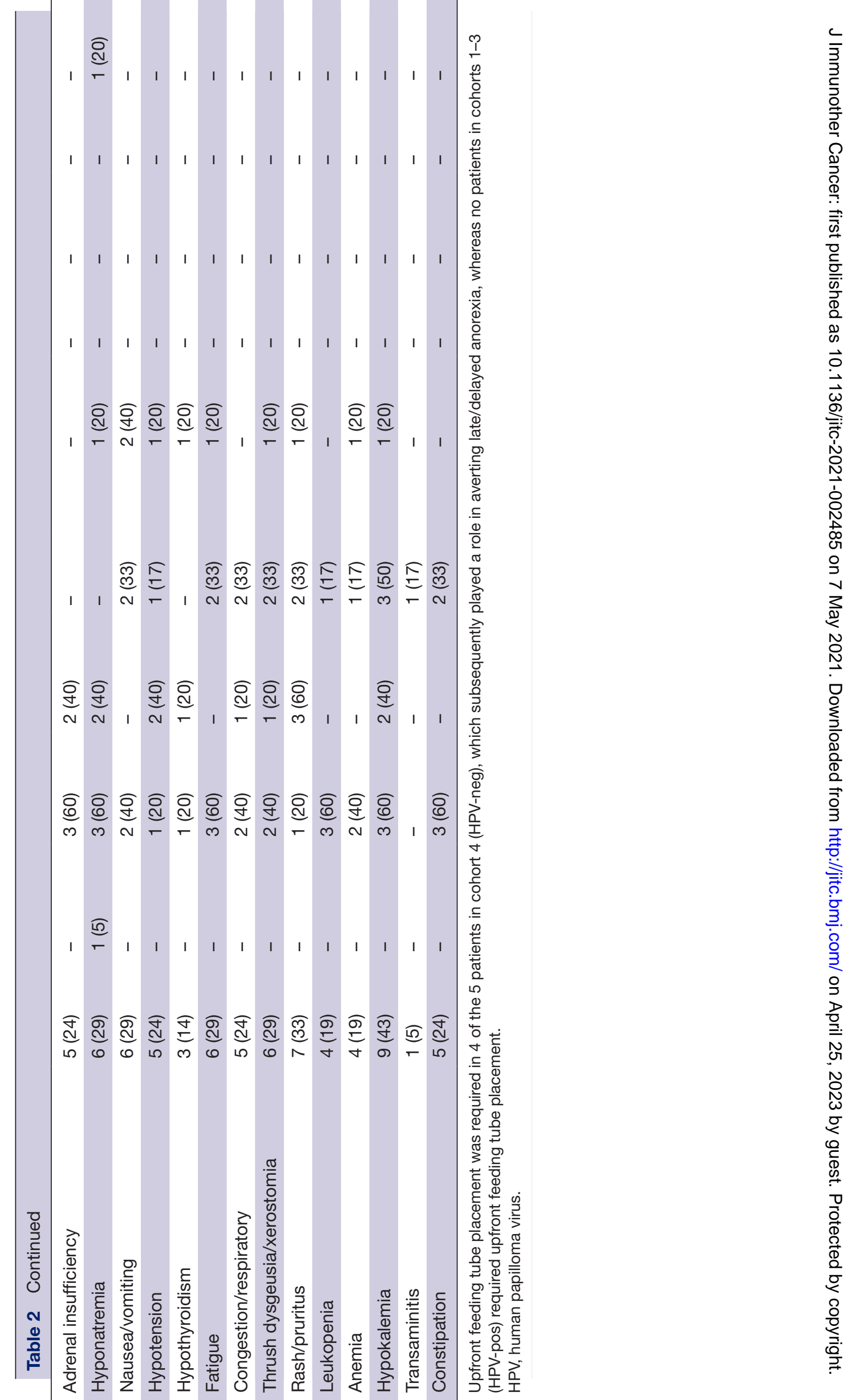


quickly and uneventfully. Another patient in cohort 4, who underwent fibular free flap reconstruction, developed exposed hardware that required removal of the plate (grade 3 Clavien-Dindo).

Delayed TRAEs occurring more than 30 days postoperatively, and considered most likely related to the combination of neoadjuvant therapy and surgery, appeared greater in the higher radiation dose cohort (cohort 1) (table 2). Grade 3 head and neck pain $(n=3)$, delayed wound healing $(n=3)$ and anorexia $(n=3)$ occurred in the three patients in cohort 1 who received mucosal radiation, which resulted in gastrostomy tube placement for each patient. Two patients in cohort 2 had grade 3 delayed wound healing; however, this was managed without the need for feeding tube placement.

The duration of these toxicities and study follow-up are summarized in figure 2A. Grade 2 adrenal insufficiency was seen in 5 patients (24\%). Patients with confirmed adrenal insufficiency were managed with corticosteroids until adrenal function returned to normal. Adrenal insufficiency lasted longer in the higher SBRT dose cohort (mean $=221$ days, range 100-330 days) compared with the lower dose cohort (mean=127 days, range 116-139 days), but all patients recovered adrenal function by 11 months. No adrenal insufficiency was seen in patients in cohort 3 who did not receive nivolumab nor was it seen in the HPV negative cohort 4 despite both cohorts being screened for endocrine abnormalities in the postoperative period. One study patient experienced a grade 4 pneumonitis related to aspiration pneumonia, which required mechanical ventilation and resolved.

\section{Efficacy outcomes}

Preoperative restaging CT scans were performed a median of 42 days (range, 38-52 days) and surgery a median of 44 days (range 40-54 days) after the first nivolumab infusion. Two patients went to surgery approximately 1 week outside of the protocol window due to patient preference and not associated with neoadjuvant treatment. Two patients failed to obtain restaging imaging. Radiographic response prior to surgery is summarized in figure 2B. Of the 21 patients, 10 patients demonstrated a partial radiologic response (PR) by RECIST criteria prior to surgery and 10 patients had stable disease (SD). No patient had a radiographic complete response; however, radiographic response was not concordant with pathological response to neoadjuvant treatment (figure 3).

Clinical to pathological down-staging occurred in $90 \%$ of the patients treated $(n=19)$. The two patients without clinical to pathological downstaging were both clinically staged as N0 and received no neck radiation, but had occult lymph node metastasis on pathological assessment of the neck dissection specimen (table 3). Among the 21 patients, $\mathrm{mPR}$ and pathological complete response (pCR) rates were $86 \%$ and $67 \%$, respectively (figure $2 \mathrm{~B}$ ). Among the $10 \mathrm{HPV}$-positive patients who underwent treatment with nivolumab and SBRT, the pCR rate was $90 \%$ (cohort $1=5 / 5$; cohort $2=4 / 5$ ) and $\mathrm{mPR}$ rate was $100 \%$. Among
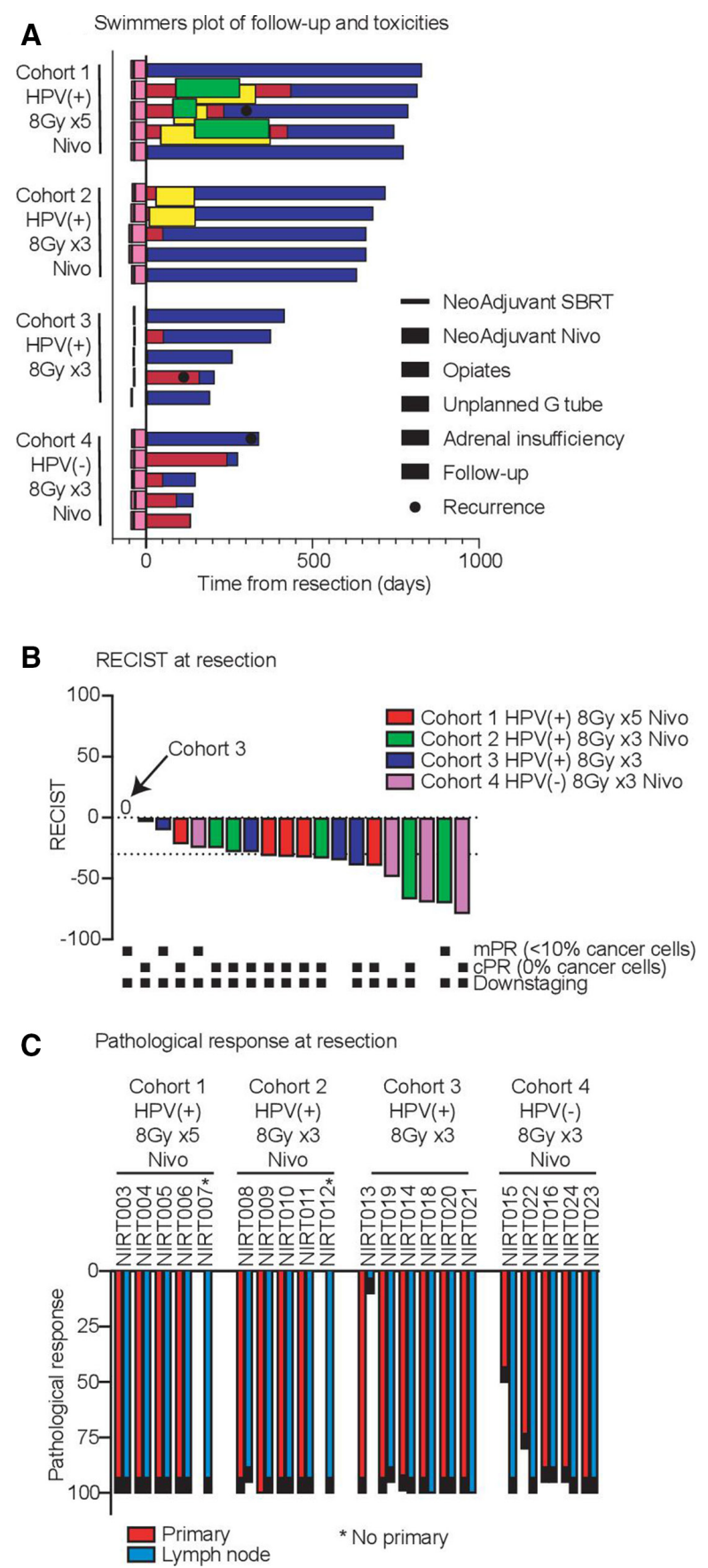

Figure 2 Toxicity swimmers plot. (A) Timing of neoadjuvant nivolumab and stereotactic body radiation therapy (SBRT) are indicated prior to resection. Extent of follow-up is shown in blue, and known recurrences are indicated. Grade 3 or greater pain requiring opiates are indicated in red. Unplanned temporary gastrostomy tube placement is indicated in green. In cohort 4, g-tubes were placed intraoperatively, per routine, to facilitate postoperative free-flap wound healing. Grade 2 or higher adrenal insufficiency requiring corticosteroid use is indicated in yellow. (B) RECIST response at surgery. The $y$-axis represents per cent tumor response, delineated by cohort and pathological response (indicated by black dots below). (C) Pathological response at surgery. The yaxis represents per cent pathological response in either the primary tumor site or metastatic lymph node, delineated by cohort. cPR, pathological complete response; HPV, human papilloma virus; mPR, major pathological response. 


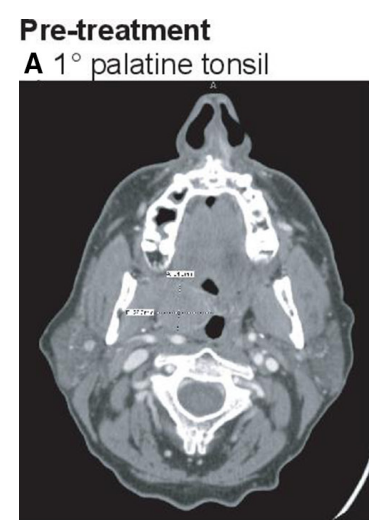

B Metastatic lymphadenopathy

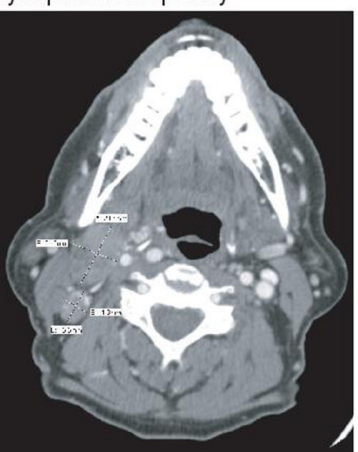

Radiation treatment

C Isodose plan to GTV +2-3mm

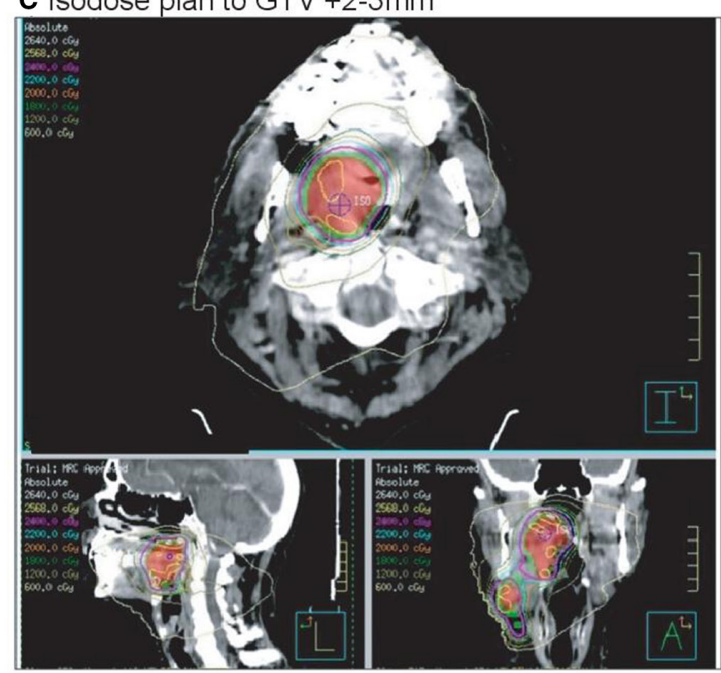

Post-treatment

D $1^{\circ}$ palatine tonsil

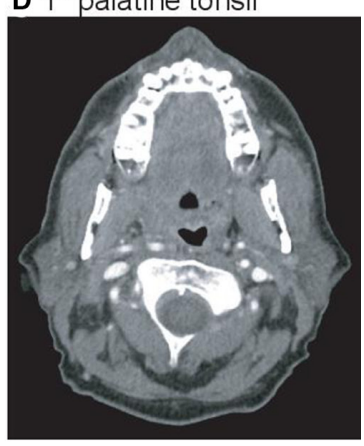

E Level II lymph nodes

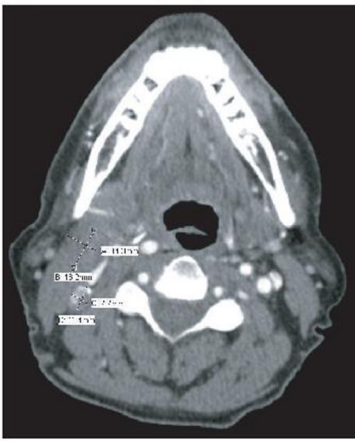

Figure 3 CT imaging of a 63-year-old man (NIRT008) with cT2N1M0 HPV+ squamous cell carcinoma (SCC) of the tonsil. (A) Pretreatment axial image demonstrating primary tumor involving the palatine tonsil. (B) Pretreatment CT demonstrating right metastatic lymphadenopathy. (C) Radiation isodose plan to GTV $+2-3 \mathrm{~mm}$. (D) Post treatment CT demonstrating partial radiographic response by RECIST $(-71 \%)$ with near complete resolution of the primary tumor. (E) Post treatment radiographic response in level II lymph nodes. Neoadjuvant treatment resulted in pathological complete response (pCR) in the primary and major pathological response (mPR) in the largest metastatic lymph node ( $<10 \%$ viable tumor cells).

HPV-positive patients treated with neoadjuvant SBRT alone (cohort 3), the pCR rate was 50\% ( $\mathrm{n}=3)$. Among HPV-negative patients (cohort 4 ), the pCR and mPR rates were $20 \%(n=1)$ and $60 \%(n=3)$, respectively.

Histopathological treatment effect and tumor regression were observed in all primary tumors and metastatic lymph nodes that were targeted with radiation. Regardless of cohort or HPV status of the resection specimen, tissue responses were characterized by a robust infiltrate of lymphocytes and macrophages into the regression bed, tumor cell necrosis, fibrosis, cholesterol clefts, and plasma cells (figure 4).

Three patients initiated neoadjuvant therapy with an unknown primary site after staging PET/CT imaging. Our approach to interrogate the patient with metastatic HPVrelated carcinoma of the cervical lymph nodes with an unknown primary is to interrogate the lingual and palatine tonsil tissue by performing bilateral tonsillectomy and base of tongue biopsies (or robotic assisted "pancake" biopsies) in an attempt to identify primary tumor. The NIRT protocol called for radiation to be delivered to GTV only, so unknown primary patients did not receive SBRT to mucosal sites. In one patient with an unknown primary (NIRT003), non-viable primary tumor was found in the un-radiated, ipsilateral tonsillectomy specimen.
Importantly, only one of the 21 patients in the study had pathological findings at surgery indicating a need for adjuvant therapy and was treated with adjuvant radiation or chemotherapy $(5 \%)$. One patient with HPV negative oropharyngeal cancer required adjuvant chemoradiation due to extensive subclinical nodal disease and a positive surgical margin; however, adjuvant radiation or chemoradiation was avoided in all other patients on study. At a median of 472 days of postoperative follow-up (range, 130-823 days), all patients were alive and 20 patients (95\%) were without evidence of disease. Two patients developed lymph node recurrence: one in the unirradiated, unoperated, contralateral neck (NIRT005) and the other in level 5 of the ipsilateral, operated neck (NIRT020). Both of these patients underwent salvage neck dissection (without adjuvant radiation) and are currently without evidence of disease. A third patient (NIRT015) with very advanced p16 negative oropharyngeal cancer recurred in the residual tongue base 1 year following near total glossectomy and adjuvant chemoradiation, refused additional surgery, and is being treated with immunotherapy with SD at the time of data cut-off. 


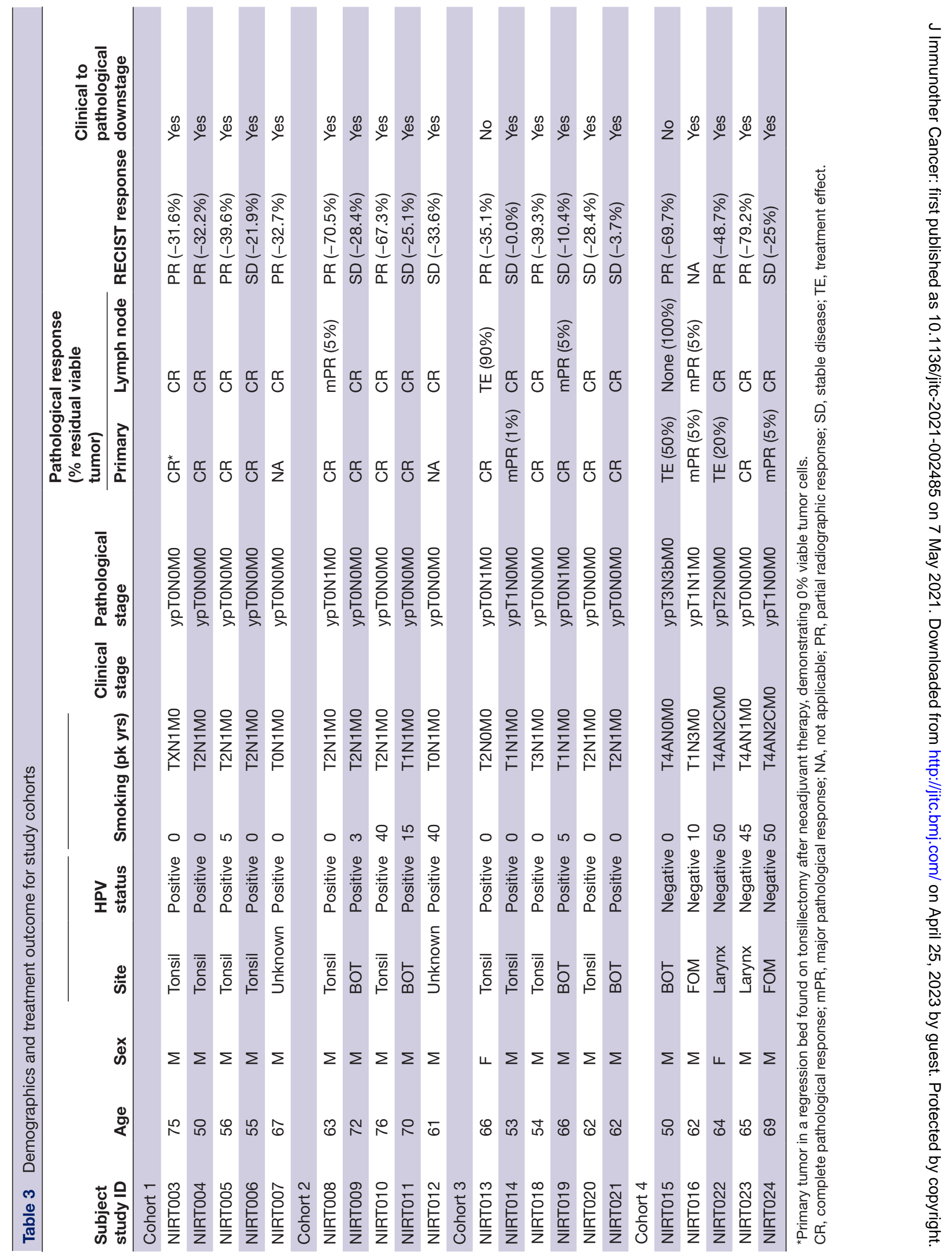


A NIRT006 LN met PCR

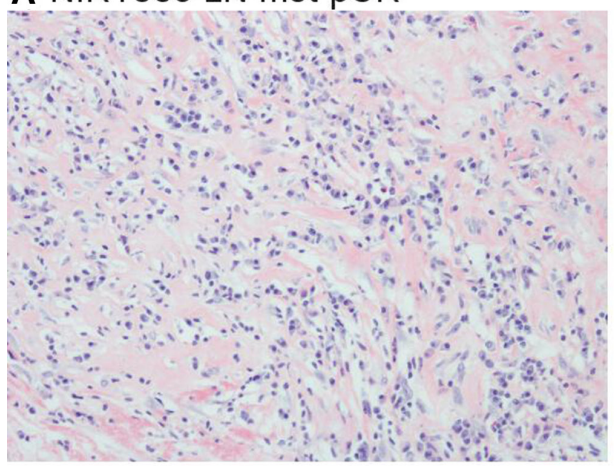

\section{NIRT008 LN met 5\% RVT}

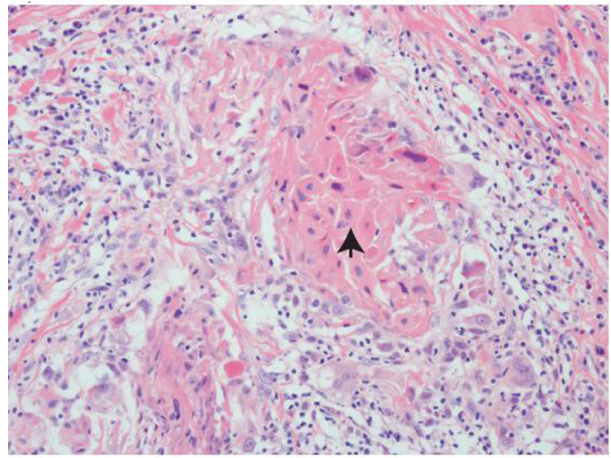

E NIRT010 $1^{\circ}$ tum

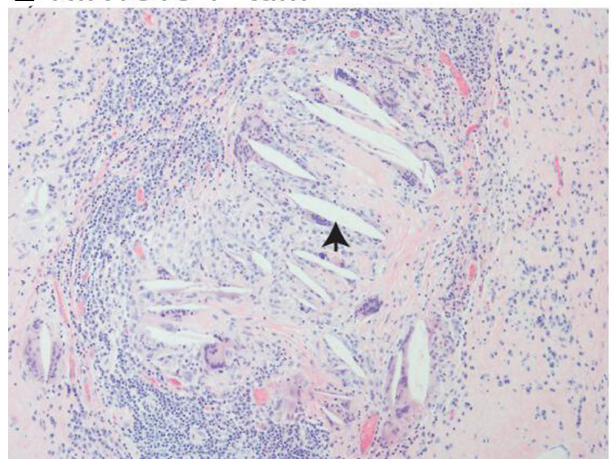

B NIRT008 $1^{\circ}$ tum PCR

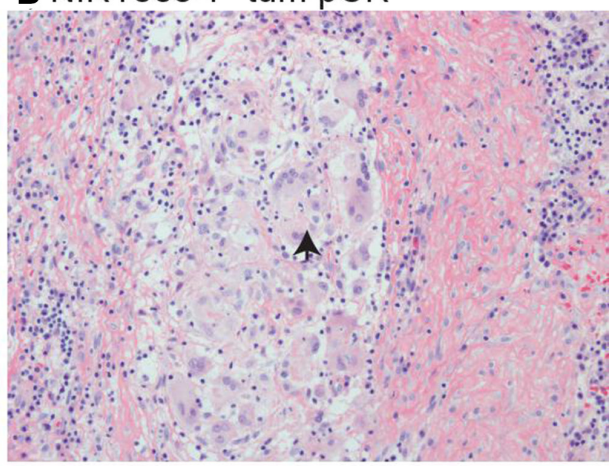

D NIRT010 $1^{\circ}$ tum pCR

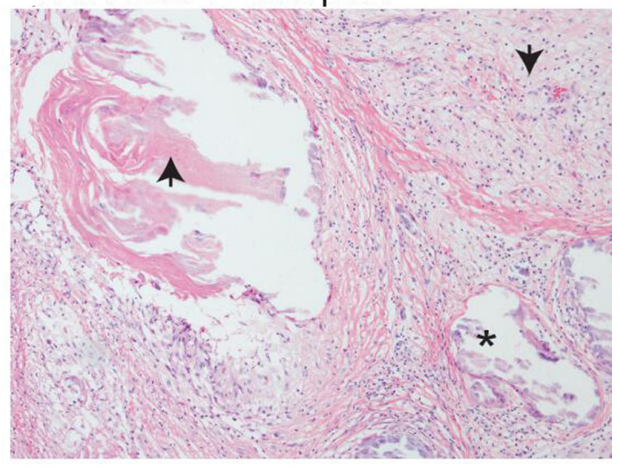

F NIRT019 LN met 5\% RVT

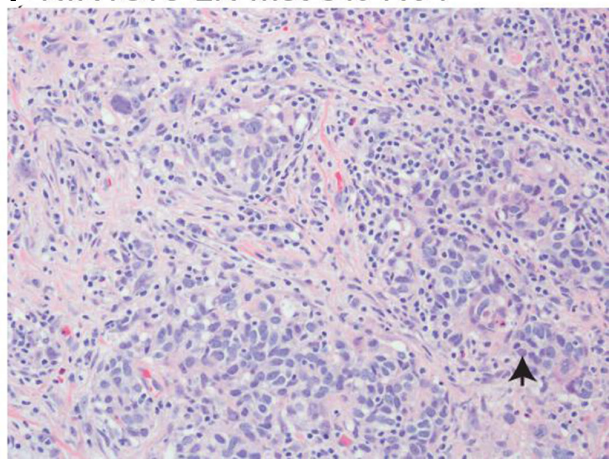

Figure 4 Representative photomicrographs of resection specimens illustrating features of pathological response in the regression bed of HPV+ subjects. (A) Fibrosis and plasma cell infiltration in metastatic lymph node (NIRT006, cohort 1) with pathologic complete response $(\mathrm{pCR})$ (magnification $\times 200$ ). (B) Giant cells (arrow) and fibrosis in primary tumor (NIRT008, cohort 2) with pCR (magnification $\times 200$ ). (C) Residual viable tumor (arrow) in metastatic lymph node (NIRT008, cohort 2, \%RVT=5) with major pathological response $(\mathrm{mPR})($ magnification $\times 200)$. (D) Necrosis (left, arrow), calcification (star) and histiocytes (right, arrow) in metastatic lymph node (NIRT010, cohort 2) with PCR (magnification $\times 100)$. (E) Cholesterol clefts (arrow) with giant cells in primary tumor (NIRT010, cohort 2) with pCR (magnification $\times 100$ ). (F) Residual viable tumor (arrow) in metastatic lymph node (NIRT019, cohort 3, \%RVT=5) with mPR (magnification $\times 200)$.

\section{DISCUSSION}

This is the first report of neoadjuvant immunoradiotherapy in the definitive setting for HNSCC and represents a potentially paradigm shifting approach to treatment of locally advanced disease by eliminating the need for 6 weeks of adjuvant radiation therapy in 20 of 21 patients and reducing the extent of surgical resection. Importantly, we observed that the combination of neoadjuvant SBRT with nivolumab was safe and resulted in clinical to pathological downstaging in $90 \%$ of patients. Although only five patients in our study had HPV-negative disease, the $80 \%(n=4)$ downstaging rate we observed in cohort four compares favorably to that of neoadjuvant pembrolizumab $(19 \%)$ and the combination of nivolumab and ipilimumab $(53 \%)$ reported in previous neoadjuvant studies in HPV negative oral cavity squamous cell carcinoma. ${ }^{78}$ In addition, the rate of mPR seen in our study $(86 \%)$, which has been described to be prognostic in melanoma, ${ }^{2}$ was significantly higher than that reported with pembrolizumab alone $(3 \%),{ }^{7}$ nivolumab alone $(7 \%)$ or in combination with ipilimumab $(20 \%) .{ }^{8}$ Additional follow-up studies will be necessary to determine whether mPR correlates with disease free survival or overall survival in HNSCC. 
It is important to note that SBRT alone was effective in generating mPR (83\%) in HPV-positive patients enrolled in this study (cohort 3). Through ongoing genomics studies of tissue and blood samples collected from patients in this trial, we are assessing whether there were transcriptional differences between the baseline biopsy and surgical resection specimens, as well as the impact that nivolumab had on various immune cell types in the tumor microenvironment. Future studies should assess the individual contribution of each modality (immunotherapy and radiation therapy) in achieving pathological responses in both HPV-positive and HPV-negative patients.

Radiologic response was a poor indicator of pathological response and did not correlate in this study. It should be noted that in this and other presurgical 'window' trials, the neoadjuvant window is relatively short ( 6 weeks in the current trial). In contrast, other non-surgical neoadjuvant trials may have a treatment window of 2-3 months, in which case RECIST is likely a meaningful indicator of clinical response. While further regression by RECIST might have been observed with a longer surgical window, our data suggest that RECIST could be misleading as a clinical endpoint in clinical trials with a short neoadjuvant treatment time prior to surgery.

The relatively modest rate of grade 3 toxicity compared with conventional chemoradiation in historical cohorts suggests that neoadjuvant immunoradiotherapy has the potential to improve quality of life in patients with HPVpositive HNSCC by reduced radiation fields and fewer treatment days. Notably, since patients in this study were treated after surgery based on standard histopathological risk features, and most patients had little if any viable tumor in either the primary site or metastatic lymph nodes, only one patient in our study met the criteria for adjuvant radiation or chemoradiation (NIRT15). This patient was clinically staged as cT4AcNOM0, had little evidence of response to neoadjuvant treatment to the primary tumor and underwent near total glossectomy and free flap reconstruction, generating a positive margin and nodal metastasis with extracapsular extension $>2 \mathrm{~mm}$ (ypT3N3bM0). None of the other patients enrolled in this study required adjuvant radiation and those without a known primary or evidence of nodal metastasis were spared radiation entirely at those sites. This is particularly important given recent preclinical evidence that elective nodal irradiation reduces the efficacy of combination stereotactic radiation therapy and immunotherapy. ${ }^{28}$ The addition of nodal irradiation attenuated chemokine expression, restrained immune infiltration, and adversely affected survival when combined with immune therapy, including PDlinhibitors, thus supporting our approach that maintains intact lymphatic drainage during therapy. Furthermore, given the significant acute morbidity associated with standard treatment options for HNSCC -6 to 7 weeks of definitive chemoradiation, or 6 weeks of adjuvant radiation \pm chemotherapy after surgery-and the critical importance of local control in HNSCC, future studies should assess health related quality of life prospectively, with validated tools, and evaluate organ preservation strategies with either watchful waiting or biopsy directed surgery in cases with complete clinical regression, especially in HPV positive tumors where the rate of mPR was high.

Two patients recurred in the neck after treatment. NIRT005 recurred in the unirradiated, unoperated, contralateral neck and was treated with salvage neck dissection alone. NIRT020 recurred in level 5 of the ipsilateral, operated neck, which was outside of the standard level 2-4 neck dissection that we perform routinely in this patient population. ${ }^{31}$ The patient was salvaged with surgical excision of level 5 lymph nodes alone and did not receive adjuvant radiation therapy. Both of these 'recurrences' likely represented occult nodal disease that was not identified by imaging or in the therapeutic neck dissection. These cases highlight the necessity for neck dissection, regardless of pathological response in the primary or metastatic node, as SBRT is delivered to GTV only, sparing the adjacent draining lymph node basins. Therefore, atypical or contralateral occult lymph node metastasis outside of the treatment field or surgically interrogated lymph node stations might be missed and should be evaluated closely in future trials.

The current study is limited by a small sample size and absence of validated endpoints to define a clinically meaningful pathological response to neoadjuvant immunotherapy. However, our trial demonstrates safety, and provides baseline pathological and radiographic response rates, as well as tissue for biologic interrogation. Ongoing studies at our institute are assessing the biopsy specimens obtained from patients in this trial before and after radiation alone or in combination with nivolumab for evidence of transcriptional changes in immune cell infiltrates and tumor cells that may provide important insights into radiation synergy with immunotherapy, biomarkers of response and/or combination strategies.

In conclusion, this study demonstrates that neoadjuvant SBRT and nivolumab is safe and results in a high rate of $\mathrm{mPR}$ in patients with HPV-positive and HPV-negative HNSCC. Since neoadjuvant SBRT alone also resulted in $\mathrm{mPR}$, future trials should compare the contribution of the individual components (SBRT and nivolumab) to pathological response.

\section{Author affiliations \\ ${ }^{1}$ Providence Cancer Institute, Portland, Oregon, USA \\ ${ }^{2}$ Earle A Chiles Research Institute, Portland, Oregon, USA \\ ${ }^{3}$ Division of Radiation Oncology, The Oregon Clinic, Portland, Oregon, USA \\ ${ }^{4}$ Department of Pathology, Providence Health and Services- Oregon, Portland, Oregon, USA \\ ${ }^{5} \mathrm{Head}$ and Neck Institute, Portland, Oregon, USA}

Twitter Rom Leidner @romleidner, Marcus A Couey @MarcusCouey and R Bryan Bell @rbryanbell

Acknowledgements The authors are grateful to the donors and supporters of Providence Portland Medical Foundation and, especially to the patients who participated in this clinical trial. 
Contributors RL, MC, KY and RBB conceived and designed the study; collected, analyzed and interpreted the data, developed figures, and wrote the report. MG performed the immune monitoring, collected, analyzed and interpreted the peripheral blood data; designed the figures; and wrote pertinent elements of the report. RL, MC, KY, RBB, AAP, AAC, ALW and SN treated patients. HX, YW and $\mathrm{CB}$ collected and analysed pathological specimens, interpreted the pathological findings and developed histology figures and legends. MC collected data and designed figures. GM and LR were involved in patient consents and data collection. All authors were involved in the critical review of the manuscript and approved the final version.

Funding National Institutes of Health, R01 CA182311 (MJG), R01 CA244142 (MJG), R01 CA208644 (MRC), Providence Portland Medical Foundation.

Competing interests The Earle A. Chiles Research Institute receives research funding from Bristol-Myers Squibb, none of which was used in the conduct of this trial; and AstraZeneca. RSL reports grants from Bristol Myers Squibb, personal fees from Merck, personal fees from Sanofi, personal fees from Regeneron, personal fees from Oncolys, outside the submitted work; MRC reports research grants from Jounce Therapeutics, grants from Nanobiotix, outside the submitted work. KHY reports non-financial support and material support for clinical trial from Eli Lilly; sponsored project agreement and institutional grant support from Bristol-Myers Squibb; and other support from AstraZeneca, outside the submitted work; CBB reports a consultant/advisory relationship with PrimeVax and Bristol-Myers Squibb; stock ownership and scientific board membership in PrimeVax and BioAl; and holds a patent on image processing systems and methods for displaying multiple images of a biological specimen that was outside the submitted work; WJU reports grants from Bristol-Myers Squibb, grants and other institutional support from Galectin Therapeutics, grants and other institutional support from Astra Zeneca, grants and other institutional support from Prometheus, grants and other institutional support from Merck, outside the submitted work; MJG reports grants from Bristol Myers Squibb, grants from Mavupharma, outside the submitted work; RBB reports grants and personal fees from Bristol Myers Squibb, grants and personal fees from Merck, personal fees from Regeneron, outside the submitted work. All other authors declare no competing interests.

\section{Patient consent for publication Not required.}

Ethics approval The protocol and its amendments were approved by the Providence Health and Services Institutional Review Board (IRB\#2017000322).

Provenance and peer review Not commissioned; externally peer reviewed.

Data availability statement Data are available upon reasonable request. All data relevant to the study are included in the article or uploaded as supplementary information. All data associated with this trial are available from Dr Bell or Research Operations Director at the Earle A. Chiles Research Institute by written request to the following address: 4805 NE Glisan St. Suite 2N35 Portland, OR 97213.

Supplemental material This content has been supplied by the author(s). It has not been vetted by BMJ Publishing Group Limited (BMJ) and may not have been peer-reviewed. Any opinions or recommendations discussed are solely those of the author(s) and are not endorsed by BMJ. BMJ disclaims all liability and responsibility arising from any reliance placed on the content. Where the content includes any translated material, BMJ does not warrant the accuracy and reliability of the translations (including but not limited to local regulations, clinical guidelines, terminology, drug names and drug dosages), and is not responsible for any error and/or omissions arising from translation and adaptation or otherwise.

Open access This is an open access article distributed in accordance with the Creative Commons Attribution Non Commercial (CC BY-NC 4.0) license, which permits others to distribute, remix, adapt, build upon this work non-commercially, and license their derivative works on different terms, provided the original work is properly cited, appropriate credit is given, any changes made indicated, and the use is non-commercial. See http://creativecommons.org/licenses/by-nc/4.0/.

\section{ORCID iDs}

Rom Leidner http://orcid.org/0000-0003-0788-7938

Michael Gough http://orcid.org/0000-0002-5575-0074

R Bryan Bell http://orcid.org/0000-0002-3775-109X

\section{REFERENCES}

1 Amaria RN, Reddy SM, Tawbi HA, et al. Neoadjuvant immune checkpoint blockade in high-risk resectable melanoma. Nat Med 2018;24:1649-54.
2 Huang AC, Orlowski RJ, Xu X, et al. A single dose of neoadjuvant PD-1 blockade predicts clinical outcomes in resectable melanoma. Nat Med 2019;25:454-61.

3 Powles T, Kockx M, Rodriguez-Vida A, et al. Clinical efficacy and biomarker analysis of neoadjuvant atezolizumab in operable urothelial carcinoma in the ABACUS trial. Nat Med 2019;25:1706-14.

4 Forde PM, Chaft JE, Smith KN, et al. Neoadjuvant PD-1 blockade in resectable lung cancer. N Engl J Med 2018;378:1976-86.

5 Chalabi M, Fanchi LF, Dijkstra KK, et al. Neoadjuvant immunotherapy leads to pathological responses in MMR-proficient and MMRdeficient early-stage colon cancers. Nat Med 2020;26:566-76.

6 Ferris RL, Gonçalves A, Baxi SS, Martens UM, et al. An open-label, multicohort, phase 1/2 study in patients with virus-associated cancers (CheckMate 358): safety and efficacy of neoadjuvant nivolumab in squamous cell carcinoma of the head and neck (SCCHN). Annals of Oncology 2017;28:v628-9.

7 Uppaluri R, Campbell KM, Egloff AM, et al. Neoadjuvant and adjuvant pembrolizumab in resectable locally advanced, human Papillomavirus-Unrelated head and neck cancer: a multicenter, phase II trial. Clin Cancer Res 2020;26:5140-52.

8 Schoenfeld JD, Hanna GJ, Jo VY, et al. Neoadjuvant nivolumab or nivolumab plus ipilimumab in untreated oral cavity squamous cell carcinoma: a phase 2 open-label randomized clinical trial. JAMA Oncol 2020;6:1563-70.

9 Ferrarotto R, Bell D, Rubin ML, et al. Impact of neoadjuvant durvalumab with or without tremelimumab on $\mathrm{CD}^{+}$tumor lymphocyte density, safety, and efficacy in patients with oropharynx cancer: CIAO trial results. Clin Cancer Res 2020;26:3211-9.

10 Gillison ML, Trotti AM, Harris J, et al. Radiotherapy plus cetuximab or cisplatin in human papillomavirus-positive oropharyngeal cancer (NRG oncology RTOG 1016): a randomised, multicentre, noninferiority trial. Lancet 2019;393:40-50.

11 Tao Y, Auperin A, Sire C, et al. Improved outcome by adding concurrent chemotherapy to cetuximab and radiotherapy for locally advanced head and neck carcinomas: results of the GORTEC 200701 phase III randomized trial. J Clin Oncol 2018:3084-90.

12 Kiyota N, Tahara M, Fujii H, et al. Phase II/III trial of post-operative chemoradiotherapy comparing 3-weekly cisplatin with Weekly cisplatin in high-risk patients with squamous cell carcinoma of head and neck (JCOG1008). J Clin Oncol 2020;38:6502.

13 Bell RB, Gough MJ, Seung SK, et al. Erratum to 'Cytoreductive surgery for head and neck squamous cell carcinoma in the new age of immunotherapy' [Oral Oncol. 61 (2016) 166-176]. Oral Oncol 2017;66:e3

14 Bernstein MB, Krishnan S, Hodge JW, et al. Immunotherapy and stereotactic ablative radiotherapy (ISABR): a curative approach? Nat Rev Clin Oncol 2016;13:516-24.

15 Curti B, Crittenden M, Seung SK, et al. Randomized phase II study of stereotactic body radiotherapy and interleukin-2 versus interleukin-2 in patients with metastatic melanoma. $J$ Immunother Cancer 2020;8:e000773.

16 Postow MA, Callahan MK, Barker CA, et al. Immunologic correlates of the abscopal effect in a patient with melanoma. $N$ Engl $J$ Med 2012;366:925-31.

17 Twyman-Saint Victor C, Rech AJ, Maity A, et al. Radiation and dual checkpoint blockade activate non-redundant immune mechanisms in cancer. Nature 2015;520:373-7.

18 McBride S, Sherman E, Tsai CJ, et al. Randomized phase II trial of nivolumab with stereotactic body radiotherapy versus nivolumab alone in metastatic head and neck squamous cell carcinoma. J Clin Oncol 2021;39:30-7.

19 Reits EA, Hodge JW, Herberts CA, et al. Radiation modulates the peptide repertoire, enhances $\mathrm{MHC}$ class I expression, and induces successful antitumor immunotherapy. J Exp Med 2006;203:1259-71.

20 Garnett CT, Palena C, Chakraborty M, et al. Sublethal irradiation of human tumor cells modulates phenotype resulting in enhanced killing by cytotoxic T lymphocytes. Cancer Res 2004;64:7985-94.

21 Chakraborty M, Abrams SI, Coleman CN, et al. External beam radiation of tumors alters phenotype of tumor cells to render them susceptible to vaccine-mediated T-cell killing. Cancer Res 2004;64:4328-37.

22 Deng L, Liang H, Burnette B, et al. Irradiation and anti-PD-L1 treatment synergistically promote antitumor immunity in mice. J Clin Invest 2014;124:687-95.

23 Crittenden MR, Zebertavage L, Kramer G, et al. Tumor cure by radiation therapy and checkpoint inhibitors depends on pre-existing immunity. Sci Rep 2018;8:7012.

24 Zebertavage LK, Alice A, Crittenden MR, Gough MJ, et al. Transcriptional upregulation of NLRC5 by radiation drives STINGand Interferon-Independent MHC-I expression on cancer cells and T cell cytotoxicity. Sci Rep 2020;10:7376. 
25 Gough MJ, Crittenden MR, Young KH. Comparing equals when evaluating immunotherapy with different doses and fractions of radiation therapy. Immunotherapy 2015;7:847-9.

26 Young $\mathrm{KH}$, Baird JR, Savage T, et al. Optimizing timing of immunotherapy improves control of tumors by Hypofractionated radiation therapy. PLoS One 2016;11:e0157164.

27 Crocenzi T, Cottam B, Newell P, et al. A hypofractionated radiation regimen avoids the lymphopenia associated with neoadjuvant chemoradiation therapy of borderline resectable and locally advanced pancreatic adenocarcinoma. J Immunother Cancer 2016;4:45.

28 Marciscano AE, Ghasemzadeh A, Nirschl TR, et al. Elective nodal irradiation attenuates the combinatorial efficacy of stereotactic radiation therapy and immunotherapy. Clin Cancer Res 2018;24:5058-71.

29 Clavien PA, Barkun J, de Oliveira ML, et al. The Clavien-Dindo classification of surgical complications: five-year experience. Ann Surg 2009;250:187-96.

30 Cottrell TR, Thompson ED, Forde PM, et al. Pathologic features of response to neoadjuvant anti-PD-1 in resected non-small-cell lung carcinoma: a proposal for quantitative immune-related pathologic response criteria (irPRC). Ann Oncol 2018;29:1853-60.

31 Viet CT, Dierks EJ, Cheng AC, et al. Transoral robotic surgery and neck dissection for HPV-positive oropharyngeal carcinoma: importance of nodal count in survival. Oral Oncol 2020;109:104770. 